\title{
Superoutburst of a New Sub-Period-Minimum Dwarf Nova CSS130418 in Hercules
}

\author{
D. Chochol ${ }^{1}$, S. Shugarov ${ }^{1,2}$, N. Katysheva ${ }^{2}$, I. Volkov ${ }^{2}$, A. Zharova ${ }^{2}$, E. Pavlenko $^{3}$, N. Pit $^{3}$, \\ R. Zvagelsky ${ }^{3}$, O. Antoniuk ${ }^{3}$, K. Antoniuk ${ }^{3}$, A. Baklanov ${ }^{3}$, N. Borisov ${ }^{4}$, M. Gabdeev ${ }^{4}$ \\ ${ }^{1}$ Astronomical Institute of the Slovak Academy of Sciences, 05960 Tatranská Lomnica, Slovakia \\ ${ }^{2}$ Sternberg Astronomical Institute of the Moscow State University, Russia \\ ${ }^{3}$ Crimean Astrophysical Observatory, Nauchny, Ukraine \\ ${ }^{4}$ Special Astrophysical Observatory of the Russian Academy of Sciences, Nizhnij Arkhyz \\ Corresponding author: chochol@ta3.sk
}

\begin{abstract}
Multicolour photometry of a new dwarf nova CSS130418 in Hercules, which underwent superoutburst on April 18, 2013, allow to classified it as a WZ Sge-type dwarf nova. The phase light curves for different stages of superoutburst are presented. The early superhumps were used to determine the orbital period $P_{\text {orb }}=64.84(1)$ minutes, which is shorter than the period minimum $\sim 78$ minutes for normal hydrogen-rich cataclysmic variables. We found the mean period of ordinary superhumps $P_{s h}=65.559(1)$ minutes. The quiescent spectrum is rich in helium, showing double peaked emission lines of H I and He I from accretion disk, so the dwarf nova is in a late stage of stellar evolution.
\end{abstract}

Keywords: cataclysmic variables - dwarf novae - SU UMa-type - WZ Sge-type - photometry - spectroscopy - individual: CSS 130418.

\section{Introduction}

Cataclysmic variables (CVs) are close binary systems with the white dwarf (WD) accreting matter from a companion which fills its Roche lobe. Due to non-zero momentum the matter creates accretion disk around the primary component.

SU UMa-type dwarf novae (DNe) are subclass of CVs which exhibits outbursts and less frequent brighter superoutbursts. The superhumps are periodic tooth shaped light variations observed during superoutbursts. Superhumps periods are a few percent longer than the orbital period. Their amplitudes at superoutburst maximum are 0.2-0.7 mag and decrease afterwards. According to the model proposed by Whitehurst (1988) and Hirose \& Osaki (1990), superhumps are caused by tidal $3: 1$ resonance in the outer parts of accretion disk, leading to the formation of an eccentric outer ring undergoing apsidal motion. Smak (2009) proposed alternative interpretation of superhumps as being due to modulated mass transfer rate resulting in periodically enhanced dissipation of the kinetic energy of the stream.

The WZ Sge-type objects are the most extreme subgroup of SU UMa-type DNe with the typical orbital periods $80-90$ minutes that exhibits only superoutbursts with amplitude 6-8 mag and a long (several years) re- currence time. One of the most remarkable signatures of WZ Sge-type objects is a presence of early superhumps, observed in the first days of superoutburst. Early superhumps have a period very close to a binary period and commonly show a double humped profile. Osaki \& Meyer (2002) suggested, that this phenomenon is manifestation of the tidal 2:1 resonance in accretion disks of binary systems with extremely low mass ratio. Resonance forms a two-armed spiral pattern of tidal dissipation, which leads to a double-peaked feature in the orbital light curve.

Very few CVs have orbital periods below the so called period minimum $\sim 78$ minutes.

\section{$2 \quad$ CSS130418}

\section{$2.1 \quad$ Discovery}

On 2013 April 18.47, the Catalina Real-Time Transient Survey (CRTS) detected an outburst of CSS130418: 174033.5+414756 in Hercules (Drake et al., 2009). The brightness of the object at the time of discovery was 14.04 mag. According to CRTS circular, the object had an 7.5 mag outburst in 2007, that lasted two month.

Prieto et al. (2013) discovered the transient independently with the All Sky Automated Survey for SuperNovae (ASAS-SN) on 2013, April 19.53, when it 
brightness was $V=12.7$ mag. They found a precursor with $B=19.2$ and $R=18.2 \mathrm{mag}$ in USNO-A2 cata$\log$ and previous outburst in GALEX UV source in the GALEX DR5 catalog with $F U V=13.62$ and $N U V=$ 14.17 mag on 2007 May 27. An optical spectrum of the transient, taken by the OSMOS imagine spectrograph of the MDM 2.4m telescope on 2013, April 21.41, shows a blue continuum (black-body $T_{\text {eff }}=16000 \mathrm{~K}$ ) with Balmer and He I lines in absorption and weak He II in emission.

\subsection{Light curve of the superoutburst}



Figure 1: $V$ light curve of superoutburst.

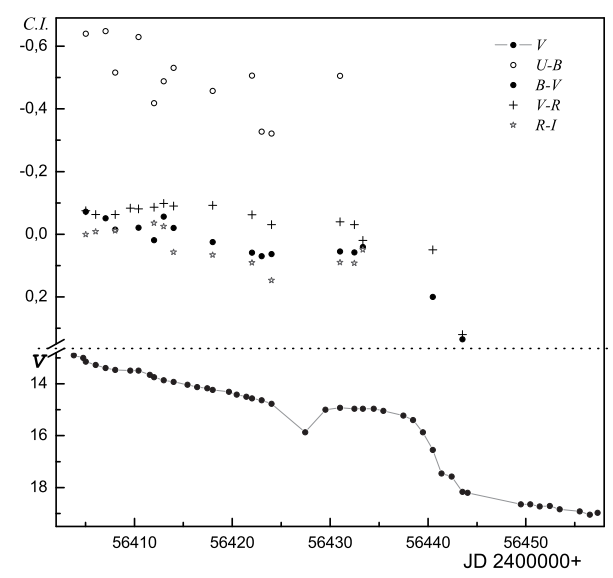

Figure 2: Mean $V$ light curve and colour indices curves of superoutburst.

Our $U B V(R I)_{C}$ CCD observations were carried out with the $0.6 \mathrm{~m}$ telescope at the Stará Lesná Observatory, $0.4,1 \mathrm{~m}$ and $1.25 \mathrm{~m}$ telescopes of the Crimean Observatory and $0.6 \mathrm{~m}$ telescope at the Crimean Laboratory of the Sternberg Astronomical Institute. We added the CCD observations of P. Dubovský taken by $1 \mathrm{~m}$ telescope at Kolonica saddle (Slovakia) available at http : //var2.astro.cz/Slovakia and AAVSO $V$ data. The superoutburst $V$ light curve is presented in Fig. 1.

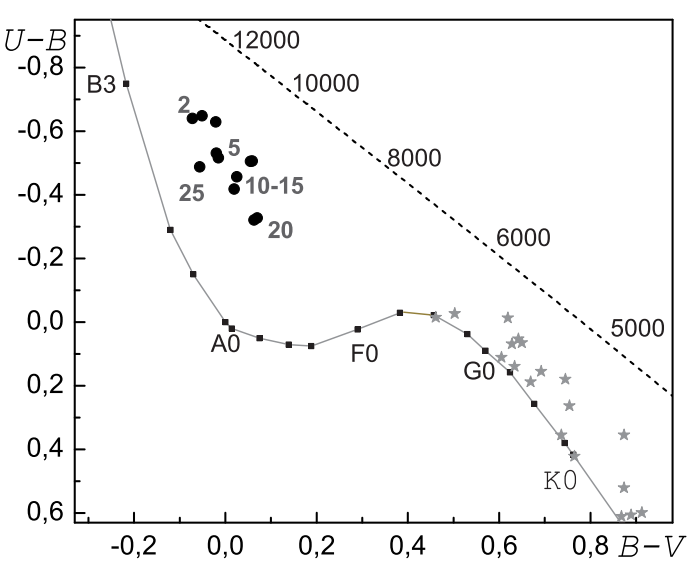

Figure 3: Two colour diagram.

The average night magnitudes of $V$ observations from Fig. 1 and our colour indices data are shown in Fig. 2.

The evolution of the nova in a two colour $(U-B, B-$ $V$ ) diagram is displayed in Fig. 3. The main and black body sequences are plotted with solid and dashed lines, respectively. The positions of field stars are marked by stars. Filled circles correspond to the positions of the dwarf nova at the days after maximum, which occurred at $\mathrm{JD}=2456403$.

\subsection{Early superhumps}

Early superhumps are periodic modulations of the brightness of WZ Sge-type dwarf novae seen during superoutburst with a period identical to the orbital period

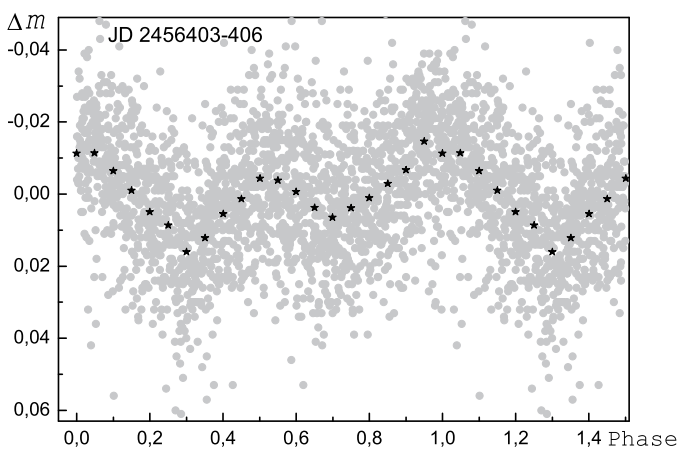

Figure 4: Phase light curve of early superhumps.

(Ishioka et al., 2002). Therefore, they are known as orbital humps. After the declining trend removal from $V$ and $B$ light curves of all available data, taken in the first 4 days after maximum, we found the ephemeris 
for brightness maxima of early superhumps by Fourier period analysis as follows:

$$
J \operatorname{Dmax}=2456402.076+0.045026 \times E .
$$

The phase light curve of early superhumps including the mean light curve is shown in Fig. 4.

\subsection{Ordinary superhumps}

Ordinary superhumps are periodic modulation seen during superoutburst after disappearance of early superhumps. Our $V$ observations of superhumps of the object, obtained during the night run 15 days after maximum (AM), are presented in Fig. 5.

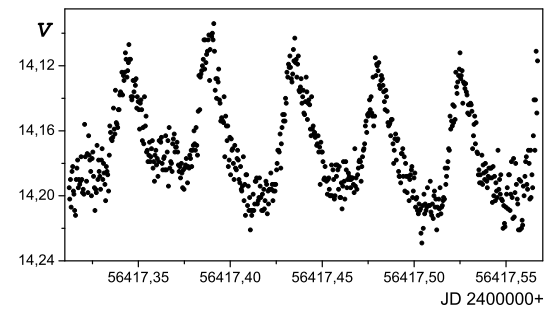

Figure 5: $V$ light curve of superhumps.

After the declining trend removal from $V$ and $B$ light curves of all available data taken on days 5-22 AM we found the mean ephemeris for brightness maxima of ordinary superhumps by Fourier period analysis as follows:

$$
J D \max =2456402.092+0.0455269 \times E .
$$

The evolution of ordinary superhumps during the superoutburst are presented in Figs. 6-10 in different days AM. The ephemeris (2) was used for all data in phase light curves.

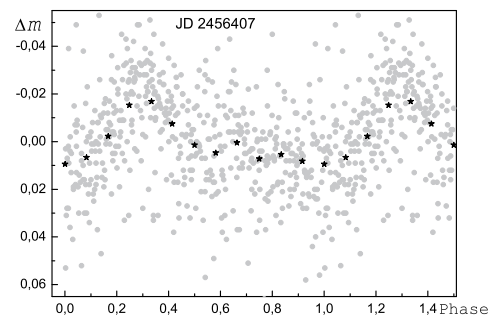

Figure 6: Ordinary superhumps on day 5 AM.



Figure 7: Ordinary superhumps on days 7-8 AM.



Figure 8: Ordinary superhumps on days 13-14 AM.

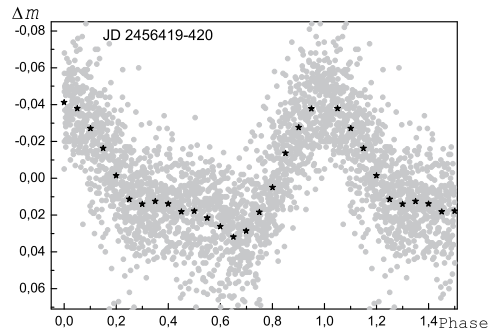

Figure 9: Ordinary superhumps on days 17-18 AM.



Figure 10: Ordinary superhumps on days 21-22 AM. 


\section{Our Spectroscopy}

Our spectra were taken with the SCORPIO spectrograph of the $6 \mathrm{~m}$ telescope in SAO during the superoutburst and quiescence of the DN in the range 4050$5100 \AA$. They are presented in Fig. 11. The spectrum during superoutburst shows H I and He I lines in absorption and He II in emission. Quiescent spectrum is rich in helium, showing double peaked emission lines of $\mathrm{H} \mathrm{I}$ and He I from accretion disk. The spectrum is similar to the spectrum of the helium-rich cataclysmic variable SBSS $1108+574$, which has the orbital period 55.3 minutes (Carter et al. 2013).

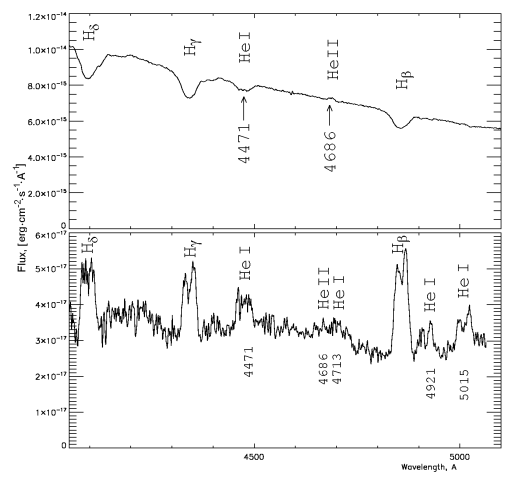

Figure 11: Spectrum of the object in outburst (May $4 / 5,2013$ ) and quiescence (August 2/3, 2013).

\section{Discussion and Conclusions}

We found a new sub-period-minimum CV with the orbital period $P_{\text {orb }}=64.84(1)$ minutes and classified it as a WZ Sge-type dwarf nova. Period of ordinary superhumps $P=65.559(1)$ minutes allowed to determine the fractional superhumps excess $\epsilon=\left[P_{\text {sh }} / P_{\text {orb }}-1\right]=$ 0.0111 (4) and also the mass ratio $q=0.0565(20)$, applying the relation of Patterson et al. (2005): $\epsilon=0.18 q$ $+0.29 q^{2}$.

Presented object CSS130418 enlarged the group of DNe under the period minimum and can help to understand its evolutionary status. Hydrogen lines in the spectrum exclude the possibility that the object belongs to AM CVn stars. The existence of early and ordinary superhumps shows that CSS130418 is evolved WZ Sge-type DN. The most probable scenario of evolution is that the mass transfer started near the end of a main-sequence lifetime of the donor star. After the donor transferred its hydrogen envelope, the mass transfer continue from hydrogen deficient interior of the star. The presence of strong He I lines in the spectrum of the object supports this view.

\section{Acknowledgement}

We acknowledge with thanks the variable star observations from the AAVSO International Database contributed by observers worldwide and used in this research. This study was supported by the VEGA grants 2/0094/11, 2/0002/13 and RFBR research projects No. 11-02-01213a, 14-02-00825 and NSh 1675.2014.2.

\section{References}

[1] Carter, P.J., Steeghs, D., de Miguel E., Goff, W., et al.: 2013, MNRAS 431, 372.

doi:10.1093/mnras/stt169

[2] Drake, A.J., Djorgovski, S.G.,Mahabal, A., Beshore, E., et al.: 2009, ApJ 696, 870. doi:10.1088/0004-637X/696/1/870

[3] Hirose, M., Osaki, Y. : 1990, Publ. Astron. Soc. Japan 42, 135.

[4] Ishioka, R., Uemura, M., Matsumoto, K., Ohashi, H., et al.: 2002, A\&A 381, L41.

[5] Osaki, Y., Meyer, F. : 2002, A\&A 383, 574.

[6] Patterson, J., Kemp, J., Harvey, D.A., Fried, R.E., et al.: 2005, PASP 117, 1204. doi:10.1086/447771

[7] Prieto, J.L., Hainline, K., Hickox, R., Goulding, A., et al. : 2013, ATel 4999.

[8] Smak, J. : 2009, AcA 59, 121.

[9] Whitehurst, R. : 1988, MNRAS 232, 35. doi:10.1093/mnras/232.1.35

\section{DISCUSSION}

CHRISTIAN KNIGGE: I think there are 3 possible interpretation for your system: 1) It could move started contact, when the donor was already nuclear evolved (like EI Psc). 2) It could be a low metallicity halo object (like J1507 = Patterson/Uthas). 3) It could be a system in which the secondary was already a substellar object before contact (as had been suggested for J1507 by Littlefair et al. (2007).

DMITRY KONONOV: How deeply evolved CVs can go beyond the period minimum?

DRAHOMÍR CHOCHOL: The shortest orbital period from already known $\mathrm{CVs}$, excluding $\mathrm{AM} \mathrm{CVn}$ stars 
(orbital periods 5 - $60 \mathrm{~min}$ ), has a dwarf nova CSS102808: 52.1 minutes (Woudt et al., 2012).

VOJTECH ŠIMON: A group of $\mathrm{CVs}$ with unexpected orbital periods of $\approx 60$ min emerging from the observations. What about the mutual similarities of the spectroscopic properties of these systems? Do they show similarities for example in the hydrogen/helium ratio?

DRAHOMÍR CHOCHOL: The HeI $\lambda 6678 / \mathrm{H} \alpha$ ratios of CSS $100603(P=65.23 \mathrm{~min})$ and SBSS $1108+574$ $(P=55.3 \mathrm{~min})$ are 1.49 and 0.81 , respectively (Breedt et al. 2012; Carter et al. 2013), so they are helium rich systems. The ratios in EI Psc and V485 Cen are higher than those typically seen in SU UMa systems. Spectrum of CSS130418 is similar to the spectra of previous objects. On the other hand in $\mathrm{J} 1507 \mathrm{HeI} \lambda 6678 / \mathrm{H} \alpha$ ratio is only 0.07 .

PAULA SZKODY: What criteria are used for calling it He-rich? Many other CVs show He I strong. Unusual short period systems have evolved donors.

DRAHOMÍR CHOCHOL: We just simply compare our spectrum with the spectrum of helium rich CV SBSS 1108+574 (Carter et al. 2013). 